\title{
ASSOCIAÇÃO ENTRE A OBESIDADE INFANTIL E A CAPACIDADE CARDIORRESPIRATÓRIA: REVISÃO SISTEMÁTICA
}

\section{Association between childhood obesity and cardiorespiratory fitness: a systematic review \\ Asociación entre la obesidad infantil y la capacidad cardiorrespiratoria: revisión sistemática}

\section{Leticia Borfe}

Universidade de Santa Cruz do Sul - UNISC - Santa Cruz do Sul (RS) - Brasil

Daiani Cristina Rech

Universidade de Santa Cruz do Sul - UNISC - Santa Cruz do Sul (RS) - Brasil

Thais Ermelinda Schulz Benelli

Universidade de Santa Cruz do Sul - UNISC - Santa Cruz do Sul (RS) - Brasil

Dulciane Nunes Paiva

Universidade de Santa Cruz do Sul - UNISC - Santa Cruz do Sul (RS) - Brasil

Hildegard Hedwig Pohl

Universidade de Santa Cruz do Sul - UNISC - Santa Cruz do Sul (RS) - Brasil

Miria Suzana Burgos

Universidade de Santa Cruz do Sul - UNISC - Santa Cruz do Sul (RS) - Brasil

\section{RESUMO}

Objetivo: Verificar as evidências científicas a respeito da associação entre obesidade infantil e aptidão cardiorrespiratória. Métodos: Realizouse uma revisão de literatura advinda da busca de artigos, publicados nos últimos cinco anos, nas bases de dados SciELO e Portal de Periódicos da CAPES. Os descritores utilizados foram aptidão fissica, sobrepeso/obesidade e crianças/adolescentes/escolares. Incluíram-se no estudo artigos completos publicados em periódicos, nos idiomas inglês, português e espanhol, com ano de publicação no período de 2010 a 2015 , e que tinham como objetivo a predição da aptidão cardiorrespiratória com testes de exercício na obesidade infantil. Resultados: Ao todo foram 97 artigos pesquisados, mas, após análise e submissão aos critérios de inclusão e exclusão, restaram cinco artigos, sendo dois em língua portuguesa, dois em espanhol e um em inglês. Dos cinco artigos analisados, quatro estudos relataram que a capacidade cardiorrespiratória foi afetada pelos índices de adiposidade corporal. Conclusão: Foi evidenciada associação inversa entre o índice de massa corporal e o condicionamento cardiorrespiratório, em que os escolares com excesso de peso apresentaram menores índices de aptidão cardiorrespiratória.

Descritores: Obesidade; Aptidão física; Crianças; Adolescentes.

\section{ABSTRACT}

Objective: To investigate the scientific evidence regarding the association between childhood obesity and cardiorespiratory fitness. Methods: A review was conducted on the literature arising from the search of articles published in the last five years in the databases SciELO and CAPES Journal Portal (Portal de Periódicos da CAPES). The descriptors used were cardiorespiratory fitness, overweight/obesity and children/adolescents/students. The study included full articles published in periodicals in English, Portuguese and Spanish, with publication year from 2010 to 2015, which aimed to predict the cardiorespiratory fitness with use of exercise testing in childhood obesity. Results: A total of 97 articles were surveyed but, after consideration and submission to the inclusion and exclusion criteria, five articles remained, two of them published in Portuguese, two in Spanish and one in English. Of the five articles analyzed, four studies reported that the cardiorespiratory fitness was affected by the body adiposity indexes. Conclusion: An inverse association was observed between the body mass index and the cardiorespiratory fitness, and the overweight students showed lower levels of cardiorespiratory fitness.

Descriptors: Obesity; Physical Fitness; Children; Adolescents. 


\section{RESUMEN}

Objetivo: Verificar las evidencias cientificas sobre la asociación entre la obesidad infantil y la aptitud cardiorrespiratoria. Métodos: Se realizó una revisión de la literatura a través de la búsqueda de artículos publicados en los últimos cinco años en la base de datos SciELO y en el Portal de Periódicos Capes. Los descriptores utilizados fueron "aptitud fisica", "sobrepeso/obesidad" y "niños/adolescentes/escolares". Se incluyeron en el estudio los artículos completos publicados en periódicos; en los idiomas inglés, portugués y español; con año de publicación entre el periodo de 2010 y 2015 y que tenían en su objetivo la predicción de la aptitud cardiorrespiratoria a través de pruebas de ejercicios para la obesidad infantil. Resultados: De los 97 artículos investigados se han quedado cinco artículos después del análisis y sumisión a los criterios de inclusión y exclusión siendo dos publicados en el idioma portugués, dos en español y uno en inglés. Cuatro de los cinco artículos analizados relataron que la capacidad cardiorrespiratoria ha sido influenciada por los indices de adiposidad corporal. Conclusión: Se evidenció la asociación inversa entre el índice de masa corporal y el acondicionamiento cardiorrespiratorio siendo los escolares con exceso de peso los que presentaron indices de aptitud cardiorrespiratoria más bajos.

Descriptores: Obesidad; Aptitud fisica; Niño; Adolescente.

\section{INTRODUÇÃO}

A obesidade é considerada uma doença multifatorial de alta prevalência entre crianças e adolescentes ${ }^{(1)}$. Um aumento de 10 a $20 \%$ na taxa de obesidade infanto-juvenil foi registrado na última década, já sendo reconhecidamente um problema de saúde pública que afeta as sociedades em diversas partes do mundo, caracterizando-se, na atualidade, como uma epidemia ${ }^{(2,3)}$. Várias doenças podem estar associadas à obesidade em crianças e adolescentes, como a hipertensão arterial sistêmica (HAS), a diabetes mellitus tipo II (DM), as doenças coronarianas, a síndrome metabólica ${ }^{(4)}$, as dislipidemias, a aterosclerose, a apneia obstrutiva do sono e hipoventilação ${ }^{(5)}$, e todas essas condições fisiopatológicas apresentam impacto sobre a qualidade e expectativa de vida dessa população( ${ }^{(6)}$.

A origem da obesidade é complexa, pois é influenciada por fatores genéticos, ambientais, biológicos, comportamentais, psicológicos e sociais ${ }^{(7)}$. O nível socioeconômico e educativo dos pais, maus hábitos alimentares, inatividade física e poucas horas de sono estão relacionados com uma maior probalidade de crianças e adolescentes serem obesos ${ }^{(4)}$. Uma evidência atual demonstra que filhos de pais obesos apresentam risco mais elevado de obesidade devido a genes e ambientes compartilhados, ou seja, aumenta o risco de uma criança se tornar um adulto obeso independente do estado de gordura na infância ${ }^{(8)}$, já que a obesidade infantil está diretamente associada à obesidade na vida adulta( ${ }^{(9)}$.

Uma boa alimentação aliada à prática de atividades físicas faz toda a diferença no peso da criança, do adolescente e também do adulto. No entanto, dados demonstram que muitas crianças e adolescentes consomem poucas frutas e legumes, e não conseguem praticar a recomendação de 60 minutos diários de atividades físicas de moderada a intensa ${ }^{(10)}$, consumindo alimentos ricos em açúcar, gordura e sal, pobres em nutrientes, envolvendo-se em atividades sedentárias com base em telas bem acima do máximo recomendado, de 120 minutos por dia. Estudo no segmento da atividade física comprovou significativas melhorias no peso de crianças que incluíram reduções do tempo de sedentarismo em frente a tela de computador e aumento de atividades físicas ${ }^{(11)}$.

Em contrapartida, a prática de atividades físicas tem sido recomendada como meio de prevenção de várias doenças, além de registrar modificações benéficas em relação a composição corporal, perfil lipídico, resistência cardiorrespiratória e diminuição da obesidade ${ }^{(12)}$. Nesse sentido, existe uma preocupação se as crianças e adolescentes estão realizando atividades físicas, tendo em vista que o nível de atividade física é fator determinante da capacidade cardiorrespiratória ${ }^{(13)}$.

Para sobreviver, o ser humano depende do oxigênio, e quando realiza um trabalho físico tem necessidade de aumentar a entrada de ar para os pulmões, incrementar a circulação sanguínea e ativar vias metabólicas específicas nos músculos esqueléticos, resultando em uma maior captação e utilização do oxigênio. Exercícios que envolvem grandes grupos musculares produzem respostas nos sistemas respiratório, cardiovascular e muscular, e essas respostas aumentam até um limite definido como consumo máximo de oxigênio $\left(\mathrm{VO}_{2 \operatorname{máx}}\right)$ ou condição aeróbica do indivíduo ${ }^{(14)}$.

A aptidão cardiorrespiratória pode ser avaliada tanto pelo limiar anaeróbico ventilatório (LAV) quanto pelo $\mathrm{VO}_{2 \max }$, apresentado de forma absoluta ou relativa ${ }^{(15)}$. $\mathrm{O} \mathrm{VO}_{2 \max }$ é considerado um importante parâmetro preditivo de morbidades associadas e um dos melhores indicadores da aptidão cardiorrespiratória ${ }^{(16,17)}$. Além de realizar o diagnóstico do nível de condicionamento cardiorrespiratório, a avaliação do $\mathrm{VO}_{2 \max }$ também é utilizada para acompanhar e prescrever o treinamento aeróbio de atletas e sedentários ${ }^{(18)}$.

Neste contexto, o presente estudo tem como objetivo verificar as evidências científicas a respeito da associação entre obesidade infantil e aptidão cardiorrespiratória. 


\section{MÉTODOS}

O presente estudo configura-se como uma revisão sistemática de estudos científicos que fizeram a relação da aptidão cardiorrespiratória com o IMC (índice de massa corpórea) de escolares. Foram consultadas as bases de dados Scientific Electronic Library Online (SciELO) e o Portal de Periódicos da Capes. Utilizaram-se os seguintes descritores para a busca dos artigos: aptidão física, sobrepeso/obesidade, criança, adolescente e escolares; tendo sido selecionados artigos publicados de janeiro de 2010 a agosto de 2015.

Para a seleção dos artigos, realizou-se, primeiramente, a leitura dos resumos das publicações encontradas, com o objetivo de refinar a amostra por meio de critérios de inclusão e exclusão. Os critérios de elegibilidade do estudo foram: artigos completos publicados em periódicos; idioma inglês, português ou espanhol; ano de publicação no período especificado de 2010 a 2015, e artigos que tinham como objetivo a predição da aptidão cardiorrespiratória com testes de exercício. Os critérios de exclusão foram: incompatibilidade do teste de aptidão cardiorrespiratória; artigos de revisão e artigos que utilizaram outras populações específicas, como adultos, idosos e portadores de necessidades especiais.

A avaliação crítica dos artigos consistiu da leitura do estudo na íntegra e, em seguida, da elaboração de quadros sinópticos com os dados coletados sobre cada pesquisa, a saber: autores/data, amostra, aspectos metodológicos e principais resultados sobre as variáveis cardiorrespiratórias e massa livre de gordura. De forma auxiliar, utilizou-se a técnica de análise temática de conteúdo por meio da leitura e releitura dos resultados dos estudos, procurando identificar aspectos relevantes que se repetiam ou se destacavam.

\section{RESULTADOS}

Foram identificados 97 estudos e desses, 84 foram excluídos após a aplicação da primeira etapa dos critérios de inclusão, resultando em 13 artigos que foram analisados na íntegra. Destes, três foram excluídos por conterem dados repetidos e estudo retrospectivo, e outros cinco foram excluídos por apresentarem incompatibilidade do teste de aptidão cardiorrespiratória e da classificação do IMC, resultando em cinco artigos para análise de revisão. A figura 1 apresenta o fluxograma da estratégia utilizada para a seleção dos artigos e demonstra o total de artigos excluídos.

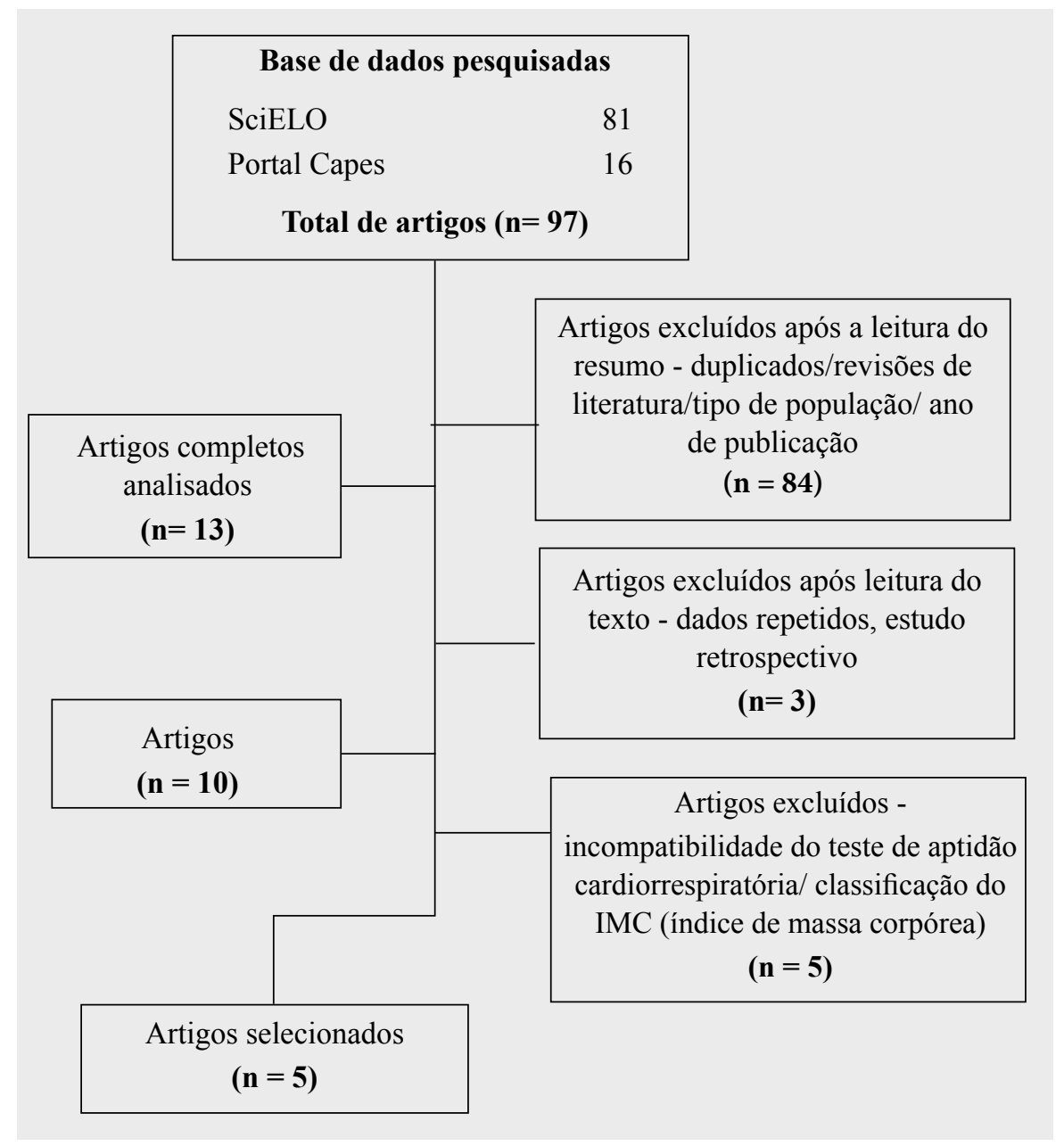

Figura 1- Fluxograma dos artigos incluídos no estudo. 
Dos artigos selecionados, dois foram publicados em língua portuguesa, dois artigos em língua espanhola e um artigo em língua inglesa. Dos cinco artigos analisados na amostra final, dois são de 2012, um é de 2013 e os outros dois artigos de 2014.

Entre os estudos selecionados, quatro adotaram a metodologia de estudo transversal e um artigo a de estudo de corte descritivo. A amostra variou de 193 a 795 escolares, totalizando 1.927 indivíduos envolvidos. Apenas um dos artigos envolveu somente estudantes do sexo feminino, os demais envolveram os escolares do sexo feminino e masculino. Os artigos envolveram uma amostra de escolares do $6^{\circ}$ ao $9^{\circ}$ ano do ensino fundamental, com uma variação de idade de 6 a 16 anos.

Os diferentes instrumentos foram classificados como diretos e indiretos, considerando-se questionários e/ou formulários como indiretos, e testes de laboratório e de campo, fatores antropométricos e exames clínicos como diretos. Três dos estudos apresentaram apenas instrumentos diretos e dois apresentaram instrumentos tanto indiretos quanto diretos.

Os artigos incluídos no estudo são apresentados no Quadro I, sendo possível observar informações sobre os autores, ano de publicação, país onde a pesquisa foi realizada, amostra, aspectos metodológicos, principais resultados sobre as variáveis cardiorrespiratórias e o excesso de peso. Os resultados mostram que a baixa capacidade cardiorrespiratória está relacionada com sobrepeso/obesidade, sendo que escolares com IMC elevado apresentaram valores menores quando comparados com seus pares eutróficos.

Quadro I - Características dos artigos analisados descritas por autor, país, amostra, aspectos metodológicos e principais resultados.

\begin{tabular}{|c|c|c|c|}
\hline Autores/País & Amostra & $\begin{array}{c}\text { Aspectos } \\
\text { metodológicos }\end{array}$ & Principais resultados \\
\hline $\begin{array}{l}\text { Santana et al. }{ }^{(13)} \\
\text { Brasil }\end{array}$ & $\begin{array}{l}417 \text { estudantes de ambos } \\
\text { os sexos com idades entre } \\
10 \text { a } 13 \text { anos }\end{array}$ & Estudo transversal & $\begin{array}{l}\text { O sobrepeso afetou negativamente a aptidão } \\
\text { cardiorrespiratória de meninos e meninas. }\end{array}$ \\
\hline $\begin{array}{l}\text { Gómes-Campos et al. }{ }^{\left({ }^{\prime}\right)} \\
\text { Peru }\end{array}$ & $\begin{array}{l}795 \text { crianças com idades } \\
\text { entre } 6 \text { a } 11 \text { anos que vivem } \\
\text { em altitude moderada }\end{array}$ & Estudo transversal & $\begin{array}{l}\text { A baixa capacidade cardiorrespiratória está } \\
\text { diretamente relacionada ao excesso de peso, } \\
\text { apresentando valores negativos fracos. }\end{array}$ \\
\hline $\begin{array}{l}\text { Galavíz et al. }{ }^{(20)} \\
\text { México }\end{array}$ & $\begin{array}{l}193 \text { escolares com idade } \\
\text { média de } 11 \text { anos }\end{array}$ & Estudo transversal & $\begin{array}{l}\text { Em ambos os sexos observou maior aptidão } \\
\text { cardiorrespiratória nos indivíduos com } \\
\text { menor IMC, Circunferência da Cintura e } \\
\text { Soma de Dobras Cutâneas. }\end{array}$ \\
\hline $\begin{array}{l}\text { Duque; Parra } \\
\text { Colômbia }\end{array}$ & $\begin{array}{l}325 \text { escolares de } 10 \text { a } 12 \\
\text { anos }\end{array}$ & Estudo transversal & $\begin{array}{l}\text { A capacidade cardiorrespiratória não } \\
\text { foi afetada pelos índices de adiposidade } \\
\text { corporal. }\end{array}$ \\
\hline $\begin{array}{l}\text { Capel et al. }{ }^{(22)} \\
\text { Brasil }\end{array}$ & $\begin{array}{l}197 \text { meninas com idades } \\
\text { entre de } 10 \text { a } 16 \text { anos }\end{array}$ & Estudo transversal & $\begin{array}{l}\text { Os maiores valores de IMC e percentual } \\
\text { de gordura corporal apresentaram menores } \\
\text { valores de } \mathrm{VO}_{2} \text { máx. }\end{array}$ \\
\hline
\end{tabular}

IMC= índice de massa corpórea; $\mathrm{VO}_{2}$ máx= consumo máximo de oxigênio.

\section{DISCUSSÃO}

Em todos os artigos destacados foi possível evidenciar que a obesidade em crianças e adolescentes afetou de forma negativa a aptidão cardiorrespiratória, sendo destacado que a associam à redução da aptidão cardiorrespiratória com indicadores de excesso de peso ${ }^{(23)}$. A aptidão física, considerada como um dos mais importantes indicadores de saúde, possui, entre seus vários componentes, a aptidão aeróbia, a qual apresenta relação inversa com os indicadores de adiposidade corporal ${ }^{(24)}$.

O aumento da capacidade aeróbica está inversamente associado ao acúmulo de gordura e outros fatores de risco cardiovascular, pois a melhoria do condicionamento aeróbico desencadeia uma série de estímulos fisiológicos que potencializam a captação de oxigênio e o uso dos ácidos graxos como fonte de energia, reduzindo os depósitos de gordura corporal e a obesidade ${ }^{(25)}$.

Diante disso, um estudo ${ }^{(22)}$ avaliou 197 meninas, com idades entre 10 e 16 anos, estudantes de duas escolas públicas do município de Atibaia, São Paulo, Brasil, com o objetivo de identificar e relacionar a composição corporal, o IMC e a idade de menarca com a capacidade aeróbica. $\mathrm{O}$ estudo observou que meninas com maiores valores de percentual de gordura e IMC apresentaram menores valores de $\mathrm{VO}_{2 \max }$. A idade de menarca em idades mais precoces e o avanço da idade cronológica são fatores importantes na redução da capacidade aeróbica, avaliada pelo $\mathrm{VO}_{2 \max }$ indireto. Além disso, a idade média de menarca foi maior nas meninas com IMC adequado quando comparadas com as meninas com sobrepeso e obesidade. 
Estudo com adolescentes ${ }^{(26)}$ apontou que o estágio maturacional é um fator relevante e que deve ser observado, porque há intima relação entre os valores de $\mathrm{VO}_{2 \max }$ e a fase puberal. Sendo assim, a avaliação do estágio maturacional pode ser mais importante do que a idade cronológica, especialmente em indivíduos obesos. Outra pesquisa ${ }^{(27)}$ revelou que os adolescentes com excesso de peso apresentam tendência a maturação mais precoce quando comparados aos não obesos.

Um estudo realizado em Recife, Pernambuco, Brasil ${ }^{(27)}$ com 417 escolares, entre 10 e 13 anos de idade, com objetivo de analisar a associação entre o estado nutricional e os componentes da aptidão física relacionada à saúde, verificou que os estudantes com sobrepeso apresentaram menor aptidão física quando comparados aos estudantes com peso normal e com baixo peso. Quanto maior o IMC, maior a adiposidade corporal e massa livre de gordura. Além disso, estudantes com sobrepeso apresentaram menor $\mathrm{VO}_{2 \max }$ quando comparados aos seus pares, com peso normal ou baixo peso. Quando ajustado pela idade,

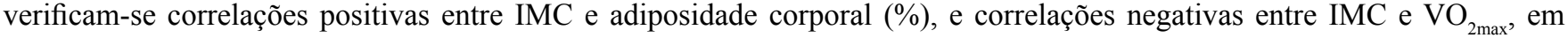
ambos os gêneros.

Valores de $\mathrm{VO}_{2 \max }$ inferiores em obesos, que poderiam limitar o consumo máximo de oxigênio, ocorrem devido a duas hipóteses: primeiramente, de que haveria uma limitação central, isto é, depende do débito cardíaco máximo e do conteúdo de oxigênio no sangue arterial; e, em segundo lugar, de que a limitação seria periférica, expressa pela diferença arteriovenosa de oxigênio e pelo metabolismo tecidual ${ }^{(28)}$.

Um estudo transversal, realizado na cidade de Guadalajara, México ${ }^{(20)}$ avaliou 193 escolares, com idade média de 11 anos, com o objetivo de avaliar a relação independente de atividade física e aptidão cardiorrespiratória com as medidas de obesidade. Os resultados sugerem que a aptidão cardiorrespiratória seja um correlato mais forte e preditor da obesidade geral e abdominal do que a atividade física.

Ao analisar as respostas do $\mathrm{VO}_{2 \max }$ relativo à massa corporal, destaca-se que os adolescentes com excesso de peso apresentam valores menores quando comparados aos grupos de peso normal, o que pode ser explicado pela maior massa corporal que os adolescentes com excesso de peso apresentam, pela baixa aptidão cardiorrespiratória característica do estado de obesidade, ou por ambas as situações ${ }^{(29)}$.

Dentro desse contexto, o fato do indivíduo obeso apresentar valores de $\mathrm{VO}_{2 \max }$ absoluto mais elevados ou semelhantes aos dos não obesos indica capacidade funcional preservada, pois a equação de Fick (que relaciona o oxigênio bombeado pelo coração e o captado pelo tecidos) revela que o coração parece fornecer oferta adequada de oxigênio para o tecido muscular ${ }^{(30)}$, entretanto, quando expressos relativos ao peso corporal, os obesos, por terem maior massa corporal, apresentam menores valores do que os não obesos ${ }^{(31-33)}$.

Estudo realizado ${ }^{(19)}$ com 795 crianças na cidade de Arequipa, Peru, objetivou determinar a capacidade cardiorrespiratória de escolares que moram em altitude moderada. Os resultados demonstraram que os escolares de ambos os sexos têm baixos níveis de aptidão cardiorrespiratória, bem como os escolares que apresentam sobrepeso e obesidade, o que corrobora com outro estudo já descrito na literatura ${ }^{(34)}$, que indica que a altitude pode afetar negativamente a capacidade aeróbica.

Uma pesquisa $^{(21)}$ realizada com 389 escolares de Manizales, Colômbia, com idades entre 10 e 12 anos, com o objetivo de estabelecer a associação entre o tempo de tela (tempo despendido em assistir televisão e utilizar o computador) e o excesso de peso e falta de condicionamento físico, não indicou diferenças antropométricas relacionadas ao maior tempo de exposição à tela, as meninas tiveram valores significativamente mais pobres de $\mathrm{VO}_{2 \max }$ que os meninos, e o teor de gordura não afetou a aptidão cardiorrespiratória máxima ${ }^{(21)}$.

O tempo gasto em comportamento sedentário tem sido associado a resultados negativos na saúde, como a composição corporal, biomarcadores de doenças cardiovasculares e metabólicas, e uma menor capacidade cardiorrespiratória ${ }^{(35)}$, sendo evidenciado que a prática regular de atividade física promove melhora na aptidão cardiorrespiratória do indivíduo, reduzindo diversos fatores de risco para o surgimento de doenças crônico-degenerativas e contribuindo para a melhoria da qualidade de $\operatorname{vida}^{(36)}$.

Uma pesquisa realizada no Chile demonstrou que, em ambos os sexos, os bons níveis de aptidão cardiorrespiratória foram associados às maiores notas em línguas. Contudo a média de sucesso acadêmico, após ajustar o tempo de tela, fez com que essa associação se tornasse não significativa. Da mesma forma, não foi observada nenhuma relação após analisar as crianças com mais horas de tempo de tela ( $\geq 2$ horas/dia). Esses achados parecem sugerir que os pais e órgãos reguladores devem minimizar os efeitos negativos do tempo de tela sobre as vidas das crianças para maximizar o efeito benéfico de hábitos saudáveis sobre o sucesso acadêmico ${ }^{(37)}$.

\section{CONCLUSÃO}

Ao analisar os estudos que compõem esta revisão, foi possível constatar uma associação inversa entre o IMC e o condicionamento cardiorrespiratório, em que os escolares com excesso de peso apresentaram menores índices de aptidão cardiorrespiratória. Muitos são os fatores que acarretam a prevalência da obesidade, destacando-se o sedentarismo e a alimentação inadequada, uma vez que a inatividade física contribui diretamente para a diminuição da capacidade cardiorrespiratória. Destaca-se, portanto, a importância de ações a serem desenvolvidas no âmbito escolar para estímulo de hábitos saudáveis entre as crianças e os adolescentes, visto que a escola torna-se uma importante aliada na promoção da saúde e prevenção de doenças. 


\section{REFERÊNCIAS}

1. Grande AJ, Silva V, Martimbianco ALC, Carvalho APV. Atividade física para prevenção e tratamento de obesidade em crianças: evidências das Coleções Cochrane. Diagn Tratamento. 2012;17(3):101-4.

2. Rossetti MB, Britto RR, Norto RC. Prevenção primária de doenças cardiovasculares na obesidade infanto-juvenil: efeito anti-inflamatório do exercício físico. Rev Bras Med Esporte. 2009;15(6):472-5.

3. Xi B, Mi J, Zhao M, Zhang T, Jia C, Li J, et al. Trends in abdominal obesity among US children and adolescents. Pediatrics. 2014;134(2):334-9.

4. Posso M, Brugulat-Guiteras P, Puig T, Mompart-Penina A, Medina-Bustos A, Alcañiz M, et al. Prevalencia y condicionantes de la obesidad em la población infantojuvenil de Cataluña, 2006-2012. Med Clín. 2014;143(11):475-83.

5. Teixeira VSS, Fonseca BCA, Pereira DM, Silva BAK, Reis FA. Avaliação do efeito da obesidade infantil e a do adolescente sobre as propriedades ventilométricas e força muscular do sistema respiratório. ConScientiae Saúde. 2009;8(1):35-40.

6. Carvalho-Ferreira JP, Cipullo MAT, Caranti DA, Masquio DCL, Andrade-Silva SG, Pisani LP, et al. Interdisciplinary lifestyle therapy improves binge eating symptoms and body image dissatisfaction in Brazilian obese adults. Trends Psychiatry Psychother. 2012;34(4):223-33.

7. Dornelles AD, Anton MC, Pizzinato A. O papel da sociedade e da família na assistência ao sobrepeso e a obesidade infantil: percepção de trabalhadores da saúde em diferentes níveis de atenção. Saúde Soc. 2014;23(4):1275-87.

8. Vale S, Santos R, Soares-Miranda L, Mota J. The relationship of cardiorespiratory fitness, birth weight and parental BMI on adolescents' obesity status. Eur J Clin Nutr. 2010;64(6): 622-7.

9. Abreú AV, Carrizo TR, Díaz EI, Velarde MS, Prado MM, Fonio MC, et al. Niveles elevados de e-selectina soluble en una población infantojuvenil con sobrepeso. Rev Argent Endocrinol Metab. 2012;49(3):119-23.

10. Leech RM, McNaughton SA, Timperio A. Clustering of children's obesity-related behaviours: associations with sociodemographic indicators. Eur J Clin Nutr. 2014;68(5):623-8.

11. Friedemann Smith C, Heneghan C, Ward A. Moving focus from weight to health. what are the components used in interventions to improve cardiovascular health in children? PLoS One. 2015;10(8):1-13.

12. Lobo H. A prevalência de fatores cardiovascular em crianças da rede de ensino público e privado de Brasília [dissertação]. Brasília: Universidade Católica de Brasília; 2012.

13. Santana CCA, Andrade LP, Gama VD, Prado WL. Associação entre estado nutricional e aptidão física relacionada à saúde em crianças. Rev Educ Fis/UEM. 2013;24(3):433-41.

14. Araújo CGS, Herdy AH, Stein R. Maximum oxygen consumption measurement: valuable biological marker in health and in sickness. Arq Bras Cardiol. 2013;100(4):51-3.

15. Gomes KB, Carletti L, Perez AJ, Rodrigues AN. Limiar anaeróbico ventilatório em adolescentes brasileiros de ambos os sexos. Rev Bras Ciênc Esporte. 2013;35(1):65-80.

16. Albouaini K, Egred M, Alahmar A. Cardiopulmonary exercise testing and its application. Postgrad Med J. 2007;83(985):67582.

17. Adekunle AE, Akintomide A. O. Gender differences in the variables of exercise treadmill test in type 2 diabetes mellitus. Ann Afr Med. 2012;11(2):96-102.

18. Milano GE, Leite N. Comparação das variáveis cardiorrespiratórias de adolescentes obesos e não obesos em esteira e bicicleta ergométrica. Rev Bras Med Esporte. 2009;15(4):251-4.

19. Gómez-Campos R, Arruda M, Almonacid-Fierro A, Holbold E, Amaral-Camargo C, Gamero D, et al. Capacidad cardiorespiratoria de niños escolares que viven a moderada altitud. Rev Chil Pediatr. 2014;85(2):188-96.

20. Galáviz KI, Tremblay MS, Colley R, Jáuregui E, López y Taylor J, Janssen I. Associations between physical activity, cardiorespiratory fitness, and obesity in Mexican children. Salud Publica Méx. 2012;54(5):463-9.

21. Duque IL, Parra JH. Exposición a pantallas, sobrepeso y desacondicionamiento físico en niños y niñas. Rev Latinoam Cienc Soc Niñez Juv. 2012;10(2):971-81.

22. Capel TL, Vaisberg M, Araújo MP, Paiva RFL, Santos JMB, Bella ZIKJD. Influência do índice de massa corpórea, porcentagem de gordura corporal e idade da menarca sobre a capacidade aeróbia $\left(\mathrm{VO}_{2 \operatorname{máx}}\right)$ de alunas do ensino fundamental. Rev Bras Ginecol Obstet. 2014;36(2):84-9. 
23. Mello JB, Ribeiro YS, Castagna A, Bergmann MLA, Bergmann GG. Baixa aptidão cardiorrespiratória está associada ao excesso de peso em crianças e adolescentes independente do sexo e da idade. Rev Bras Ciênc Movim. 2013;21(4):56-2.

24. Silva DAS, Nascimento TBR, Silva AF, Glaner MF. Excesso de adiposidade corporal em adolescentes: associação com fatores sociodemográficos e aptidão física. Motriz. 2013;19(1):114-25.

25. Saavedra JM, Escalante Y, Garcia-Hermoso A. Improvement of aerobic fitness in obese children: a meta-analysis. Int J Pediatr Obes. 2011;6(3-4):169-77.

26. Geithner CA, Thomis MA, Vanden Eynde B, Maes HH, Loos RJ, Peeters M, et al. Growth in Peak Aerobic Power during Adolescence. Med Sci Sports Exerc. 2004;36(4):1616-24.

27. Wang Y. Is obesity associated with early sexual maturation? A comparison of the Association in American Boys Versus Girls. Pediatrics. 2002;110(5):903-10.

28. Souza F, Navarro AC, Stancati J Filho, Serra MM, Alonso AC. Respostas cardiorespiratórias de individuos sedentarios obesos e não obesos em esteira ergométrica. RBPFEX. 2014;8(44):164-71.

29. Gomes KB, Carletti L, Perez AJ. Desempenho em teste cardiopulmonar de adolescentes: peso normal e excesso de peso. Rev Bras Med Esporte. 2014;20(3):195-9.

30. Ekelund U, Franks PW, Wareham NJ, Aman J. Oxygen uptakes adjusted for body composition in normal-weight and obese adolescents. Obes Res. 2004;12(3):513-20.

31. Loftin M, Sothern M, Warren B, Udall J. Comparison and VO2 peak during treadmill and cycle ergometry in severely overweight youth. J Sports Sci Med. 2004;3(4):254-60.

32. Marinov B, Kostianev S, Turnoska T. Ventilatory efficiency and rate of perceived exertion in obese and nonobese performing standardized exercise. Clin Physiol Funct Imaging. 2002;22(4):254-60.

33. Goran M, Fields DA, Hunter GR, Herd SL, Weinsier RL. Total body fat does not influence maximal aerobic capacity. Int J Obes Relat Metab Disord. 2000;24(7):841-8.

34. Bates T, Mockler J, Dobrosielski DA. Effects of altitude on step performance [resumo]. International J Exercise Science: Conference Proceedings [Internet]. 2015 [acesso em 20 Set 16];9(3). Disponível em: http://digitalcommons.wku.edu/ ijesab/vol9/iss3/10/

35. Santos A, Andaki ACR, Amorim PRS, Mendes EL. Fatores associados ao comportamento sedentário em escolares de 9-12 anos de idade. Motriz. 2013;19(3 Supl):25-34.

36. Burgos MS, Reuter CP, Burgos LT, Pohl HH, Paiva DN, Reuter EM, et al. Aptidão cardiorrespiratória e fatores de risco cardiovasculares: um estudo com escolares de Santa Cruz do Sul, RS, Brasil. Rev Epidemiol Controle Infecç. 2013;3(4): $148-52$.

37. Aguilar MM, Vergara FA, Velásquez EJ, Marina R, García-Hermoso A. Screen time impairs the relationship between physical fitness and academic attainment in children. J Pediatr. 2015;91(4):339-45.

\section{Endereço para correspondência:}

Leticia Borfe

Avenida Independência, 2293

Bairro: Universitário

CEP 96816-501- Santa Cruz do Sul - RS - Brasil

E-mail: borfe.leticia@gmail.com 